\title{
Irreversible Binding of Zomepirac to Plasma Protein In Vitro and In Vivo
}

\author{
Philip C. Smith, Antony F. McDonagh, and Leslie Z. Benet \\ Department of Pharmacy, School of Pharmacy, and Liver Center, School of Medicine, University of California, \\ San Francisco, California 94143
}

\begin{abstract}
Zomepirac is a nonsteroidal anti-inflammatory drug recently withdrawn from use because of an unexplained high incidence of immunological reactions. It is metabolized in humans to a reactive, unstable acyl glucuronide which accumulates in plasma. Because of the similarity of zomepirac glucuronide to bilirubin glucuronide in structure and stability and the documented irreversible binding of bilirubin to albumin through its acyl glucuronide, we studied the reaction of zomepirac acyl glucuronide with albumin in vitro from pH 5 to 9 and in vivo in six healthy human volunteers who had received a single 100 -mg oral dose of zomepirac. Irreversible binding of zomepirac to protein was determined by exhaustive washing of protein, followed by hydrolysis of bound zomepirac-protein adduct with base, extraction of the liberated drug, and chromatographic measurement. Irreversible binding was observed both in vitro and in vivo. The extent of binding in vitro was time- and $\mathrm{pH}$-dependent. In vitro drug binding was also observed for the isomers of zomepirac glucuronide which were formed by intramolecular acyl migration. Irreversible binding in vivo correlated with overall exposure to zomepirac glucuronide when exposure was expressed as the area under the plasma concentration vs. time curve. When probenecid (500 $\mathrm{mg}$, twice daily), which decreases the plasma clearance of zomepirac glucuronide, was administered concurrently with zomepirac, irreversible binding of zomepirac was increased. The nature of the zomepirac protein binding is probably covalent. Formation of irreversibly protein-bound zomepirac occurs via the acyl glucuronide as previously shown for bilirubin glucuronide, and the reaction may be general for other drugs that are metabolized to acyl glucuronides.
\end{abstract}

\section{Introduction}

The nonsteroidal anti-inflammatory drug (NSAID), ${ }^{1}$ zomepirac, 5-(4-chlorobenzoyl)-1,4-dimethyl-1 $H$-pyrrole-2-acetic acid (Zomax), was withdrawn from distribution in the United States and Britain in March 1983 because of unexplained anaphylactic reactions which led to several reported deaths $(1,2)$. Zomepirac

Address correspondence and reprint requests to Dr. Benet, Dept. of Pharmacy, School of Pharmacy, 926-S, University of California, San Francisco, CA 94143.

Received for publication 4 April 1985 and in revised form 18 November 1985.

1. Abbreviations used in this paper: AUC, area under the plasma concentration vs. time curve; HPLC, high performance liquid chromatography; HSA, human serum albumin; NSAID, nonsteroidal anti-inflammatory drug; Z, zomepirac; ZG, zomepirac glucuronide.

J. Clin. Invest.

(C) The American Society for Clinical Investigation, Inc. 0021-9738/86/03/0934/06 \$1.00

Volume 77, March 1986, 934-939
$(Z)$ is representative of a large and growing group of NSAIDs of the aryl alkanoic acid class (3) which include tolmetin, indomethacin, diflunisal, fenoprofen, and ibuprofen (4). Ibuprofen has recently been approved for over-the-counter use in the United States. Because of the toxicity of $\mathrm{Z}$, as well as that of benoxaprofen (Oraflex), this group of drugs has come under increasing scrutiny by both the public and government (5). With an estimated six million arthritics and 30 million persons with other chronic joint diseases in the United States (6), the market for anti-inflammatory and analgesic drugs is large and sales of these medications amount to a billion dollars per year (7). The growth of this market and the exposure of the public to NSAIDs is likely to continue with many new drug entities being developed or introduced.

The well-documented toxicities of NSAIDs, i.e., gastric irritation, nephritis, and acute renal failure (8), were not responsible for the withdrawal of $\mathrm{Z}$ from the market. Instead, a high incidence of immunologic and anaphylactic reactions, which may be unique to $\mathrm{Z}$ and other NSAIDs, led to its withdrawal (2). Not unique to $\mathrm{Z}$ or unusual for NSAIDs of this class is their elimination by metabolic conjugation of the ubiquitous acid side chain with glucuronic acid to form an acyl glucuronide. This metabolic pathway is the major route of elimination for $\mathrm{Z}$, with up to $90 \%$ of a ${ }^{14} \mathrm{C}$-labeled dose excreted in the urine as zomepirac glucuronide (ZG) (3). This metabolite (Fig. 1) is unstable in mild alkaline solutions, and undergoes rearrangement to positional isomers and hydrolysis to $Z$ (9). Another wellknown but unrelated compound that is metabolized to unstable acyl glucuronides is bilirubin. In cholestatic liver disease, bilirubin becomes irreversibly (probably covalently) bound to albumin (10-15), and recent studies have shown that this reaction occurs via acyl glucuronide metabolites of bilirubin $(16,17)$. Although bilirubin glucuronides accumulate in human plasma only during liver disease (15), the glucuronide of zomepirac (ZG) reaches a plasma level similar to that of $\mathrm{Z}$ after administration of a single oral dose of $Z$ to healthy subjects $(3,18)$. Since both bilirubin and $\mathrm{Z}$ yield similar reactive and unstable acyl glucuronide metabolites (9), it seemed possible to us that covalent binding of $\mathrm{Z}$ to protein might occur in vivo via the $\mathrm{Z}$ glucuronide metabolite. In this paper we present evidence for formation of drug-protein adducts in human volunteers given $\mathrm{Z}$ orally.

\section{Methods}

Zomepirac, ${ }^{14} \mathrm{C}$-labeled zomepirac $(4.08 \mathrm{mCi} / \mathrm{mmol})$ and Zomax, 100 mg tablets, were obtained from McNeil Pharmaceuticals (Springhouse, PA) as the dihydrate of the sodium salt. ZG was purified from human urine (before withdrawal of the drug) (9). Purified isomeric conjugates of ZG, with $\mathrm{Z}$ on the 2, 3, or 4 positions of glucuronic acid (Fig. 1), were isolated by preparative high performance liquid chromatography (HPLC) (9). All concentrations of ZG and its isomers are given as $\mathrm{Z}$ equivalents. Human plasma was collected from volunteers into heparinized $(10 \mathrm{U} /$ $\mathrm{ml}$ ) tubes. Human serum albumin (HSA) (fraction V) and $\beta$-glucuronidase (bovine liver, Type B-10) were purchased from Sigma Chemical 


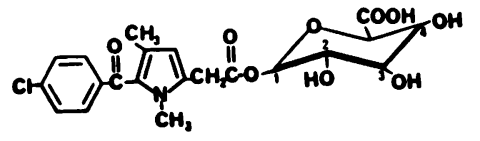

Figure 1. Structure of zomepirac $\beta$-1 glucuronide.

Co. (St. Louis, MO). Solvents used for analysis were HPLC grade, and all other chemicals were reagent grade.

Protein was measured with the Bradford method (Bio-Rad Laboratories, Richmond, CA) using HSA as the standard. Binding of $\mathrm{Z}$ to protein in vivo is expressed as nanograms of $Z$ bound per milligram of protein. In vitro binding was normalized to the amount of conjugate or $\mathrm{Z}$ present at the start of the incubation.

${ }^{14} \mathrm{C}$-labeled ZG was prepared biosynthetically as follows: $\left[{ }^{14} \mathrm{C}\right] \mathrm{Z}(23$ $\mathrm{mg}, 322 \mu \mathrm{Ci} / \mathrm{mmol}$ in $2 \mathrm{ml} 40 \%$ propylene glycol) was administered intravenously to a male rhesus monkey $(\sim 8 \mathrm{~kg})$. Spontaneously voided urine was collected at intervals through $120 \mathrm{~h}$ into a vessel containing 1 $\mathrm{ml} 86 \%$ phosphoric acid to decrease the $\mathrm{pH}$ and prevent loss of $\mathrm{ZG}$ by hydrolysis (9). Samples were then adjusted to $\mathrm{pH} 2-4$ with phosphoric acid and stored at $-20^{\circ} \mathrm{C}$.

The $0-11$-h urine sample $(360 \mathrm{ml})$, which contained $\sim 80 \%$ of the radioactivity excreted in the urine over $120 \mathrm{~h}$, was adjusted to $\mathrm{pH} 2$, washed twice with $200 \mathrm{ml}$ hexane, then extracted four times with 150 $\mathrm{ml}$ ethyl acetate. The ethyl acetate extract, which contained $80 \%$ of the radioactivity in the sample, was dried with magnesium sulfate, filtered, and evaporated to dryness with a rotary evaporator. The residue was mixed with $4 \mathrm{ml}$ of $25 \%$ acetonitrile/ $0.05 \mathrm{M}$ sodium acetate, $\mathrm{pH} 4.5$, and centrifuged to remove undissolved material. $\left[{ }^{14} \mathrm{C}\right] \mathrm{ZG}$ was isolated from the supernatant solution by preparative HPLC (9). Fractions containing ZG from successive preparative HPLC injections were combined, adjusted to $\mathrm{pH} 2$ with phosphoric acid, and evaporated under reduced pressure to remove methanol. The residual aqueous solution was extracted with ethyl acetate and the extract was dried with magnesium sulfate and evaporated to dryness. The residue was dissolved in dry methanol to give a concentrated solution of purified ZG $(\sim 189 \mu \mathrm{g} / \mathrm{ml})$, which was kept at $-20^{\circ} \mathrm{C}$ until used for binding studies; $85 \%$ of the radioactivity of this solution was shown by analytical HPLC to be present as $\left[{ }^{14} \mathrm{C}\right] \mathrm{ZG}$.

Duplicate incubations of radiolabeled $\mathrm{Z}$ and $\mathrm{ZG}$ were conducted at a concentration of $30 \mu \mathrm{g} / \mathrm{ml}(\mathrm{Z}$ equivalents, $63,000 \mathrm{cpm} / \mathrm{ml})$ with 0.5 $\mathrm{mM}(3 \mathrm{~g} / 100 \mathrm{ml}) \mathrm{HSA}$ in $0.15 \mathrm{M}$ phosphoric acid, $\mathrm{pH} 7.5$, at $37^{\circ} \mathrm{C} .1-$ $\mathrm{ml}$ aliquots, taken after $2 \mathrm{~min}, 1,6$, and $20 \mathrm{~h}$ of incubation were added to $3 \mathrm{ml}$ acetonitrile to precipitate protein and centrifuged. The protein pellet was washed 10 times with $3 \mathrm{ml}$ methanol/ether (3:1) to remove reversibly bound $\mathrm{Z}$ and conjugates. The washed protein pellet was digested with $2 \mathrm{ml}$ of $1 \mathrm{M} \mathrm{NaOH}$ for $1 \mathrm{~h}$ at $50^{\circ} \mathrm{C}$, neutralized with $0.5 \mathrm{ml}$ glacial acetic acid, and suspended in $15 \mathrm{ml}$ scintillation fluid (Aquasol; New England Nuclear, Boston, MA) for counting. Irreversible binding is expressed as counts per minute per milligram of protein, or as the fraction of the total radioactivity added to $1 \mathrm{ml}$ of the incubation which became irreversibly bound.

Further binding studies were performed with unlabeled compounds. $\mathrm{Z}, \mathrm{ZG}$, or $\mathrm{ZG}$ isomers were incubated at $37^{\circ} \mathrm{C}$ with human plasma or $0.5 \mathrm{mM}(3 \mathrm{~g} / 100 \mathrm{ml}) \mathrm{HSA}$ in $0.15 \mathrm{M}$ potassium phosphate buffer. The HSA solutions were adjusted to the desired final $\mathrm{pH}$ with dilute $\mathrm{NaOH}$ or $\mathrm{HCl}$. Z, ZG, and ZG isomers were dissolved in ethanol before use, and aliquots were added to HSA solutions to provide concentrations of $20-50 \mu \mathrm{g} / \mathrm{ml}(0.068-0.17 \mathrm{mM})$. For this experiment, mixtures of isomeric conjugates of ZG were prepared by preincubation of ZG in phosphate buffer, pH 7.4 , at $37^{\circ} \mathrm{C}$ for $90 \mathrm{~min}(9)$ followed by addition of 2,000 $\mathrm{U} / \mathrm{ml} \beta$-glucuronidase and further incubation for $15 \mathrm{~min}$. Isomeric conjugates of ZG are resistant to hydrolysis by $\beta$-glucuronidase, and HPLC analysis of the incubation solutions showed that no $Z G$ was present when the binding of the ZG isomeric conjugates to albumin was studied in vitro.

Incubation reactions were stopped by precipitating the proteins by sequential addition of 1 vol cold ethanol, $2 \mathrm{vol}$ acetonitrile, and $0.01 \mathrm{vol}$ concentrated phosphoric acid to obtain a pH of 2-4. After centrifugation $(2,250 \mathrm{~g})$ for $10 \mathrm{~min}$, the supernatant was analyzed by HPLC using a method developed in our laboratory which minimizes degradation of the labile glucuronides (19). The extent of covalent binding of $Z$ to HSA was quantified using an established method as follows (20): the protein pellet obtained after centrifugation above was washed exhaustively (10 times) with $6 \mathrm{vol}$ methanol/ether (3:1) to remove residual reversibly bound $\mathrm{Z}$ and conjugates. The washed protein was then treated with 1 $\mathrm{ml}$ of $1 \mathrm{M} \mathrm{KOH}$ at $80^{\circ} \mathrm{C}$ for $1 \mathrm{~h}$; liberated $\mathrm{Z}$ was quantitated by HPLC after acidification $\left(95 \mu 186 \% \mathrm{H}_{3} \mathrm{PO}_{4}\right)$ and extraction of the protein digest with $5 \mathrm{ml}$ dichloromethane. A standard curve was constructed by spiking blank precipitated plasma or HSA with Z prior to base-catalyzed hydrolysis. The internal standard used for HPLC quantitation was an analogue of $Z$ which has been employed in previous work (9). Zomepirac is stable under the basic conditions employed, and control studies with both ${ }^{14} \mathrm{C}$ labeled and unlabeled $\mathrm{Z}$ showed that the exhaustive washing procedure completely removed adsorbed and reversibly bound $\mathrm{Z}$ from plasma or HSA protein precipitates. The Z-protein adduct was stable under the conditions used to wash the protein, with $\sim 2.5 \%$ of adduct lost during the washing procedure, as estimated from 24-h stability studies conducted in methanol/ether (3:1). Drug not removed from plasma protein by the washing procedure but liberated only on treatment with strong base is defined in this paper as "irreversibly bound" $(20)$.

A clinical study of the effect of probenecid on $\mathrm{Z}$ disposition was conducted in our laboratory in September 1982 (18). Plasma samples from this study were examined 18 mo later for covalently bound Zprotein adduct. In this study (18), an oral dose (100 mg) of Zomax had been given to six healthy male volunteers, ages $21-30$ yr. Probenecid ( $500 \mathrm{mg}$ twice a day) was given three days before and concurrently with a second $Z$ dose to examine its effect on $Z$ and $Z G$ disposition. Plasma and urine samples taken from 0 to $48 \mathrm{~h}$ after each $\mathrm{Z}$ dose were assayed by HPLC within 2 wk for $Z$ and ZG concentrations (19). To minimize artifactual ZG loss before analysis, plasma and urine were adjusted to pH 2-4 with phosphoric acid, then frozen at $-20^{\circ} \mathrm{C}$ until analyzed. 6 of the original 12 sets of plasma samples from the 1982 study along with similarly treated control plasma samples that had been spiked with 2 $\mu \mathrm{g} / \mathrm{ml} \mathrm{ZG}$ were analyzed retrospectively, $18 \mathrm{mo}$ after collection, for irreversible binding of $\mathrm{Z}$ using the methods described above for the in vitro studies.

\section{Results}

A preliminary study measuring the $\left[{ }^{14} \mathrm{C}\right] \mathrm{Z}$ which becomes irreversibly bound to albumin after $4 \mathrm{~h}$ of incubation with [ $\left.{ }^{14} \mathrm{C}\right] \mathrm{ZG}$ indicated that incubations with $\left[{ }^{14} \mathrm{C}\right] \mathrm{ZG}$ produced 57 -fold higher binding of radioactivity to albumin than the control incubations with $\left[{ }^{14} \mathrm{C}\right] \mathrm{Z}$. Further in vitro studies examined the time-dependence of binding when $\left[{ }^{14} \mathrm{C}\right] \mathrm{ZG}$ was incubated with albumin in solution at $\mathrm{pH} 7.4,37^{\circ} \mathrm{C}$. The concentration of $\mathrm{Z}, \mathrm{ZG}$ and isomers of ZG in the supernatant after the first precipitation of albumin were measured by HPLC. Binding of radiolabel upon incubation of $\left[{ }^{14} \mathrm{C}\right] \mathrm{ZG}$ with albumin was time-dependent with an apparent plateau or maximum achieved after $6 \mathrm{~h}$, while control incubations with $\left[{ }^{14} \mathrm{C}\right] \mathrm{Z}$ produced no time-dependency in irreversible binding. The rate of binding appeared to be temporally related to the concentration of $\beta-1$ ZG present (vs. the $\mathrm{Z}$ concentration), as determined by HPLC. This finding provided strong preliminary evidence for a chemical reaction between $\mathrm{ZG}$, but not $\mathrm{Z}$, and albumin. The absolute proportion of counts which became irreversibly bound to protein by $6 \mathrm{~h}$ was $3.8 \%$ of the amount of $\left[{ }^{14} \mathrm{C}\right] \mathrm{ZG}$ added. Since the radiochemical purity of the $\left[{ }^{14} \mathrm{C}\right] \mathrm{ZG}$ used was $85 \%$, these findings did not exclude the possibility that the irreversibly bound isotope might have been derived from impurities in the $\left[{ }^{14} \mathrm{C}\right] \mathrm{ZG}$. This difficulty, coupled with limitations in the availability of $\left[{ }^{14} \mathrm{C}\right] \mathrm{Z}$ and $\left[{ }^{14} \mathrm{C}\right] \mathrm{ZG}$, prompted us to develop an alternative more specific "cold" procedure for estimating irreversible binding of $Z$ to albumin. In 
this method, "irreversibly" bound drug is defined as that liberated from the isolated washed protein by saponification and then extracted and identified by HPLC.

Irreversible binding of $\mathrm{Z}$ to protein occurred when either ZG or a mixture of its isomeric conjugates was incubated with HSA or plasma at physiologic $\mathrm{pH}$ with apparent linearity of binding over the range of concentrations employed in vitro. From replicate experiments, such as that shown in Fig. 2, binding was time-dependent and reached a plateau after $3 \mathrm{~h}$. The relative fraction of the total drug bound was $60 \%$ higher with $\mathrm{ZG}$ than with the mixture of ZG positional isomers. Control incubations with $\mathrm{Z}$ produced no detectable binding. Analyses carried out after 12 and $24 \mathrm{~h}$ of incubation revealed a slight decrease in the concentration of bound drug, presumably because of slow hydrolysis of the Z-protein adduct. The rate and extent of binding was $\mathrm{pH}$ dependent, as shown in Fig. 3. The formation rate of Z-protein adduct increased when the $\mathrm{pH}$ was raised from 5 to 9 , but the conjugates eventually rearranged by acyl migration, with increased hydrolysis occurring at the higher $\mathrm{pH}(9)$. The Z-protein adduct also became hydrolyzed at the higher $\mathrm{pH}$ values. Further experiments conducted with Z-HSA adduct at pH 7.4 and 10.0 (data not presented), as well as the use of $1 \mathrm{M} \mathrm{KOH}$ to hydrolyze the Z-protein adduct, document the labile nature of the adduct under basic conditions. However, control studies established that the Z-albumin adduct was not substantially degraded during the washing procedure. A $14 \%$ reduction in protein adduct was detected after $24 \mathrm{~h}$ in methanol/ether, which suggests that only $2.5 \%$ of the bound drug would be lost during the $4 \mathrm{~h}$ required to exhaustively wash the protein, assuming degradation to be a first order process. When the supernatants obtained from the in vitro incubations were analyzed by HPLC, the concentration of ZG, as expected (9), decreased with time, with production of isomeric conjugates of ZG and eventual slow hydrolysis to $\mathrm{Z}$. Thus, for $\mathrm{ZG}$, hydrolysis and acyl migration occur simultaneously with covalent adduct formation.

To determine the extent to which isomeric conjugates of ZG could lead to binding of $Z$ to protein, the individual purified isomeric conjugates were incubated at $\mathrm{pH} 7.4$ with HSA (Fig. 4). Incubation solutions were sampled frequently during the first hour before appreciable rearrangement to the other isomeric

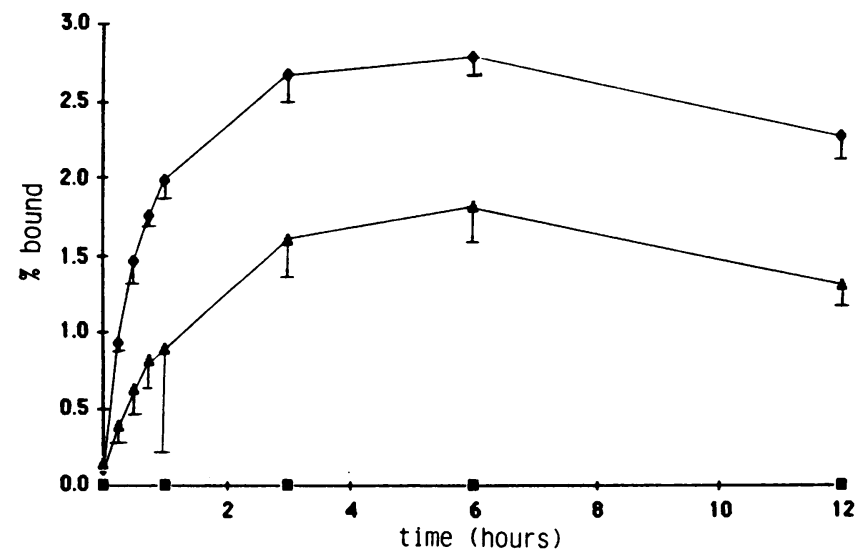

Figure 2. Irreversible binding of $Z$ to plasma protein in vitro. $Z \beta-1$ glucuronide, $\bullet$; isomeric $Z G s, \Delta$; or $Z$, $n$, were incubated with human plasma at $\mathrm{pH} 7.4$ and $37^{\circ} \mathrm{C}$ in duplicate and irreversibly bound $\mathrm{Z}$ was measured as described in Methods. (Similar results were obtained in replicate experiments in which mixtures were analyzed after different preincubation times) (Mean \pm SD).

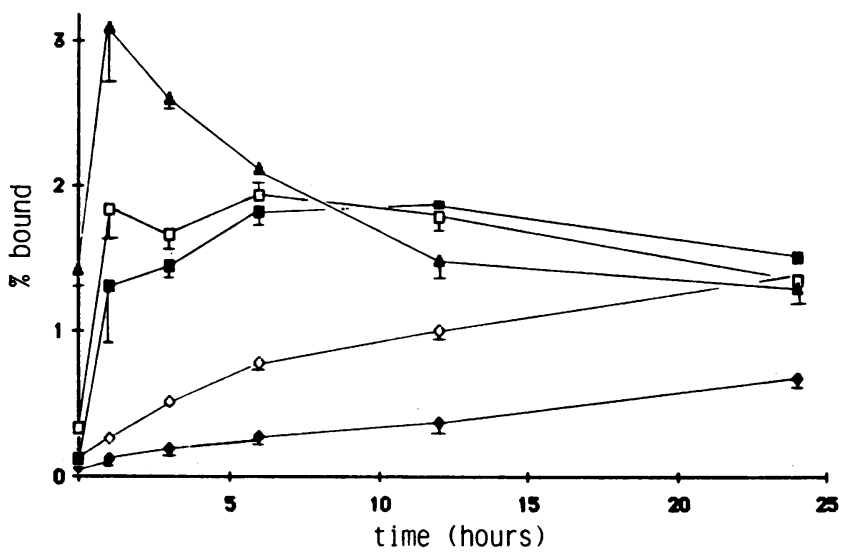

Figure 3. Influence of $\mathrm{pH}$ on the irreversible binding of $\mathrm{Z}$ to HSA in vitro. $\mathrm{Z} \beta$-1 glucuronide was incubated in duplicate at the designated $\mathrm{pH}$ with $0.5 \mathrm{M}$ HSA in $0.15 \mathrm{M}$ phosphate buffer at $37^{\circ} \mathrm{C}$ and aliquots were taken for determination of irreversibly bound $\mathrm{Z}$ (mean $\pm \mathrm{SD}$ ) ४,

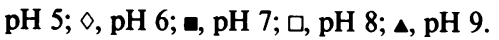

conjugates could occur. Because the available quantities of purified isomers were limited, these incubations were only done in a single experiment in duplicate. Irreversible binding was observed with each isomer, but at different rates. Therefore, $\mathrm{ZG}$ and all of its isomers formed by acyl migration are capable of leading to formation of Z-protein adduct in vitro.

These in vitro studies suggested that a similar protein adduct might be formed in vivo, as with bilirubin glucuronides $(16,17)$, if $\mathrm{ZG}$ were to persist in plasma for a sufficient length of time. In 6 volunteers given $100 \mathrm{mg} \mathrm{Z}$ orally, the molar concentrations of ZG detected in plasma were similar to $Z$ levels. The total area-under-the-plasma-concentration vs. time curve (AUC) of $\mathrm{ZG}$ and $\mathrm{Z}$ averaged $(n=6) 180 \pm 57$ and $164 \pm 64 \mu \mathrm{g}-\mathrm{min} / \mathrm{ml}$, respectively. (All AUC and concentration measurements are expressed in $Z$ equivalents.) The average peak concentrations after the $\mathrm{Z}$ dose were $1.09 \pm 0.42$ and $1.60 \pm 1.74 \mu \mathrm{g} / \mathrm{ml}$ for $\mathrm{ZG}$ and $\mathrm{Z}$, respectively. When probenecid was administered concurrently with $\mathrm{Z}$, the plasma clearance of both $\mathrm{ZG}$ and $\mathrm{Z}$ decreased dramatically, resulting in increased plasma concentrations of both. This increase was reflected by increased AUC's to $499 \pm 134$ and $708 \pm 308 \mu \mathrm{g}-\mathrm{min} / \mathrm{ml}$ for $\mathrm{ZG}$ and $\mathrm{Z}$, respectively, which represent

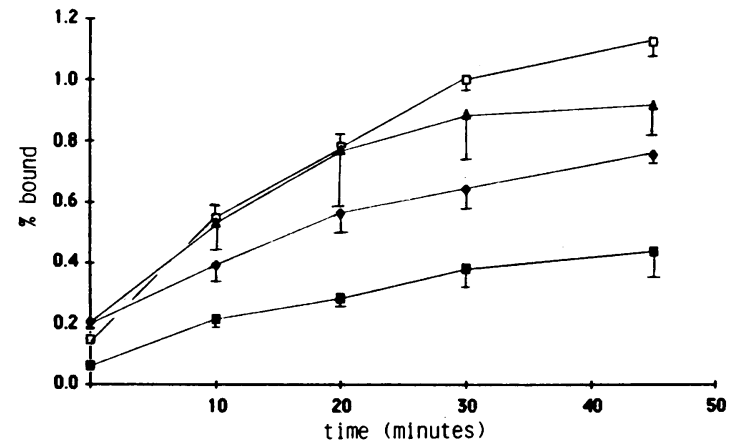

Figure 4. Irreversible binding of $\mathrm{Z}$ to HSA through the purified isomeric conjugates of ZG. Purified isomeric conjugates of $\mathrm{ZG}$ were incubated in vitro with HSA $(0.5 \mathrm{mM})$ in $0.15 \mathrm{M}$ phosphate buffer, $\mathrm{pH}$ $7.4,37^{\circ} \mathrm{C}$ in duplicate and samples were removed for analysis of $\mathrm{Z}$ protein binding. $\square, \mathrm{ZG} ; \wedge, \alpha / \beta$-2-O-acyl ZG; $\bullet, \alpha / \beta$-4-O-acyl ZG; n, $\alpha / \beta-3-O-a c y l ~ Z G$. 
2.8- and 4.3-fold increases over the values observed without probenecid. The data summarized here are presented in detail elsewhere (18).

Z-protein adduct was detected in plasma from all subjects given a single oral dose of $Z$. For each subject the extent of binding was apparently linearly related to the cumulative AUC (0-time point) of $\mathrm{ZG}$. A representative plot of this relationship is shown in Fig. 5. Superimposed on the plot (right ordinate) are concentrations of ZG measured at each time point $(t)$ corresponding to a particular cumulative $\mathrm{AUC}(0-t)$. At zero time no binding of $Z$ was detectable. Subsequently irreversible binding increased with increasing AUC $(0-t)$ until a maximum was reached when all of the reversibly bound $\mathrm{Z}$ and $\mathrm{ZG}$ in plasma had been cleared. The maximum amount of drug bound in all subjects was observed between 8 and $12 \mathrm{~h}$, which corresponded to the period during which $\mathrm{ZG}$ concentrations in plasma approached zero. Thereafter, as in the in vitro experiments, the amount of bound $\mathrm{Z}$ decreased gradually with an estimated first order half-life of $70 \mathrm{~h}$. Control plasma samples (adjusted to $\mathrm{pH}$ 2-4 and frozen at $-20^{\circ} \mathrm{C}$ ) that had been previously spiked with $2 \mu \mathrm{g} / \mathrm{ml} \mathrm{ZG}$ (which is twice the average maximum concentration of ZG found in vivo after a single 100-mg oral dose [18]), had low concentrations of Z-protein adduct $(0.30 \pm 0.07 \mathrm{ng} \mathrm{Z} / \mathrm{mg}$ protein, $n=6$ ) that were much lower than the maximum amounts detected in vivo (Fig. 6). These control data indicate that the covalent binding measured in the human study samples did not occur during storage.

As shown in Fig. 6, the concentration of irreversibly bound drug increased linearly with AUC $(0-t)$ of $\mathrm{ZG}$ for all subjects. Concurrent administration of probenecid led to an elevated total AUC of ZG and increased Z-protein adduct formation, as shown by the closed symbols in Fig. 6 .

\section{Discussion}

This work documents for the first time irreversible drug binding to albumin in vivo in humans through an acyl glucuronide metabolite. The in vitro data presented support the hypothesis that $\mathrm{ZG}$ is a precursor of adduct formation, and that $\mathrm{Z}$ itself does not react chemically with albumin. The linear correlation ob-

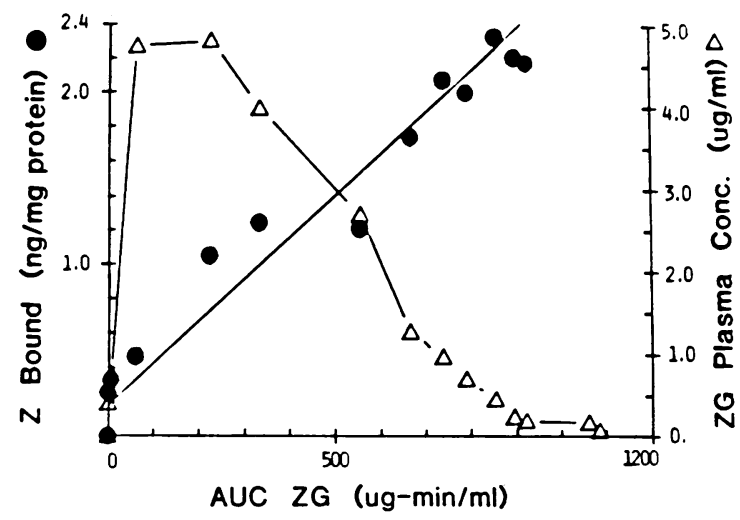

Figure 5. Irreversible binding of $\mathrm{Z}$ to plasma protein in vivo in a representative human subject. Plasma samples collected up to $48 \mathrm{~h}$ after a 100 -mg oral dose of $Z$ were analyzed for $Z$ irreversibly bound to protein, $\bullet$, (left ordinate) as described in Methods, and for ZG plasma concentration, $\triangle$, (right ordinate), by HPLC. Fitted regression from 0 to $12 \mathrm{~h}$ after the dose, $(r=0.975 ; P<0.001)$.

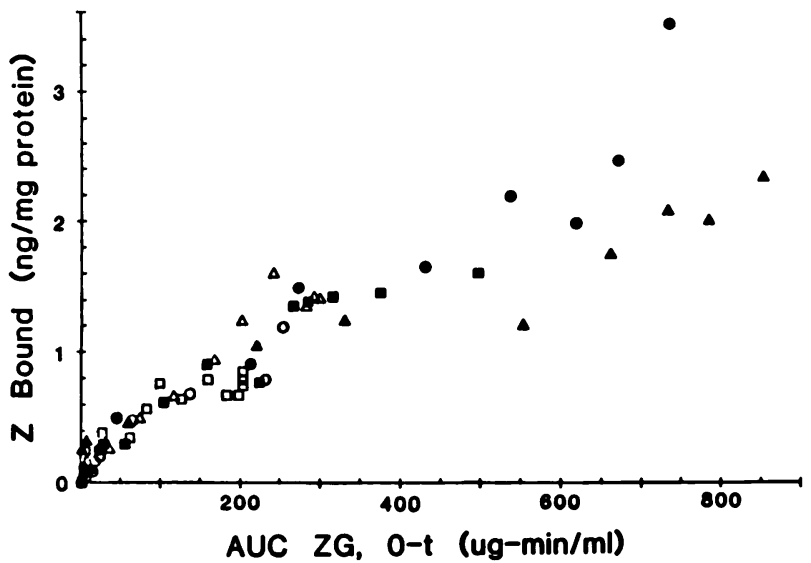

Figure 6. Irreversible binding of $\mathrm{Z}$ to plasma protein in six humans. $\mathrm{Z}$ bound irreversibly to plasma protein was determined in plasma samples collected up to $48 \mathrm{~h}$ after administration of an oral $\mathrm{Z}$ dose (100 $\mathrm{mg}$ ) to three volunteers taking probenecid (closed symbols) and three volunteers not receiving probenecid (open symbols). The data presented are from zero time to the time when binding reached a maximum at $8-12 \mathrm{~h}$ after the dose.

served between exposure to $\mathrm{ZG}$ and the degree of $\mathrm{Z}$ binding in vivo (Figs. 5 and 6 ) also supports this hypothesis. When probenecid was given concurrently, it decreased the renal clearance of $Z G$, thus increasing exposure to $Z G$ with a resultant increase in formation of irreversibly bound $\mathrm{Z}$ (Fig. 6, closed symbols). Preliminary experiments in rats and guinea pigs (data not presented) revealed negligible $Z$ binding to protein $6 \mathrm{~h}$ after an intravenous dose. In these species little or no $\mathrm{ZG}$ is detectable in plasma after an intravenous dose. Therefore, the amount of covalent binding observed clearly depends on both the concentration and residence time of the glucuronide in plasma.

The plasma concentration of irreversibly bound $\mathrm{Z}$ in man was highest 8-12 h after the drug was administered, then decreased slowly until it averaged $65 \%$ of the maximum by $24-48$ h. This slow disappearance of Z-protein adduct, which was seen also during the incubations of $\mathrm{ZG}$ with albumin in vitro, is probably due to slow hydrolytic release of $Z$ from the adduct. The irreversible binding of $Z$ via its acyl glucuronide metabolite may act as a reservoir for $\mathrm{Z}$ in the body that is depleted very slowly. Although the amount of covalently bound drug detected in the present studies represented only a small fraction of the single dose administered, it seems likely that upon repeated, multiple doses, bound drug would accumulate to a greater extent and eventually represent a larger fraction of the drug in the body or in a particular organ. Furthermore, it might be expected that disease states or advanced age, which compromise the excretion of ZG, would lead to increased ZG levels and increased adduct formation, as with bilirubin (15). Irreversible binding of bilirubin to albumin, however, is not detectable in healthy individuals (15), because bilirubin conjugates do not normally accumulate in the blood, whereas Z-protein binding was detected readily in this study in healthy young male volunteers given only a single oral dose.

Although ZG is the only glucuronic acid ester of $\mathrm{Z}$ formed biosynthetically in vivo, we studied the reactivity of isomeric conjugates of ZG because ZG does isomerize to some extent in humans in vivo (18) and in biological fluids in vitro. Adduct formation was observed in vitro when mixtures of isomers (Fig. 
2) or the individual purified components (Fig. 4) were incubated with albumin. Irreversible binding via the purified isomers was evident even during the first $20 \mathrm{~min}$, when rearrangement to other isomers was negligible and $\beta-1 \mathrm{ZG}$ was not detectable by HPLC. Thus, all of the isomers of ZG appear to react with albumin in vitro to give adducts. Presently the mechanism of the reaction is unknown. Stogniew and Fenselau (21) speculated that reaction of thiols with the electrophilic carbonyl of clofibryl glucuronide might be responsible for some of the toxicity observed for clofibrate. This laboratory also reported irreversible binding of ${ }^{14} \mathrm{C}$-label to albumin and possibly nuclear material when radiolabeled acyl glucuronides of indomethacin, flufenamic acid, and clofibric acid were incubated with the respective macromolecules (22). However, the mechanism of irreversible binding of an acid to albumin through its acyl glucuronide, as proposed by Fenselau $(21,22)$ and discussed in a recent review by Faed (23), where nucleophilic substitution at the electrophilic carbonyl displaces glucuronic acid, does not readily explain why isomeric conjugates of ZG and bilirubin glucuronides (17) also react irreversibly with albumin. An alternative mechanistic hypothesis is that acyl conjugates bind to albumin, after or concurrent with acyl migration, by formation of an imine (Schiff's base) between the 1-position of glucuronic acid and a lysine residue of albumin. There is much precedent for this latter mechanism, as glycosylation of hemoglobin (24-26) and albumin $(27,28)$ through imine formation and subsequent Amadori rearrangement have been thoroughly described.

$\mathrm{Z}$ (Zomax) was withdrawn from the market because of a high incidence of immunologic reactions which included anaphylaxis $(1,2)$. As yet, no relationship can be established between the irreversible binding of $Z$ to protein as found in these studies and the incidence of immunological reactions to $Z$ observed clinically in humans. However, it is generally accepted that immunogenic sensitizing ability and the ability to form adducts with proteins are correlated (29), although such a relationship is often difficult to prove in humans. The studies presented here show that $\mathrm{Z}$ becomes bound to albumin and plasma proteins. It is unlikely that this reactivity of ZG is unique to albumin, and formation of adducts with other tissue proteins, leading to potential haptens, might be expected in vivo.

These studies were initiated because of the similarities between ZG and bilirubin acyl glucuronides and the previous observation that bilirubin binds irreversibly to albumin via its acyl glucuronide. The phenomenon is probably a general one which occurs with other acyl glucuronides of drugs or endogenous compounds. Conjugation of acidic compounds with glucuronic acid is a major route of metabolism for many xenobiotics and represents the primary route of elimination for aryl alkanoic acid NSAIDs. As a class of drugs, the NSAIDs do require concern for serious toxicity. Interestingly, Z, benoxaprofen, ibufenac, alclofenac and indoprofen were among the 25 drugs listed in a recent review of drugs discontinued because of their toxicity (30). All five are NSAIDs of the aryl-alkyl acid class that are eliminated as acyl glucuronides.

\section{Acknowledgments}

We thank Martha Barba and Susana Atwood for typing the manuscript and Diana Fedorchak for editorial assistance.

This work was supported in part by a grant from McNeil Pharmaceuticals, Springhouse, PA, and by National Institute of Health grants
AM26307, AM26743, and GM36633. Dr. Smith was a recipient of the Merck Fellowship from the American Foundation for Pharmaceutical Education.

\section{References}

1. Samuel, S. A. 1981. Apparent anaphylactic reaction to zomepirac (Zomax ${ }^{\oplus}$. N. Engl. J. Med. 304:978.

2. Committee on Government Operations, House of Representatives, 98th Congress. April 26 and 27, 1983. FDA's Regulation of Zomax. Washington, DC. 1-555.

3. O'Neill, P. J., K. A. Yorgey, N. L. Renzi, R. L. Williams, and L. Z. Benet. 1982. Disposition of zomepirac sodium in man. J. Clin. Pharmacol. 22:470-476.

4. Hucker, H. B., K. C. Kwan, and D. E. Duggan. 1980. Pharmacokinetics and metabolism of non-steroidal anti-inflammatory agents. In Progress in Drug Metabolism. J. W. Bridges and L. F. Chasseaud, editors. John Wiley and Sons, New York. 165-253.

5. Committee on Government Operations, House of Representatives 97th Congress. Aug. 3 and 4, 1982. The regulation of new drugs by the Food and Drug Administration: the new drug review process. Washington, DC. 1-688.

6. New York Arthritis Foundation. 1976. Arthritis foundation annual report. New York. 1-16.

7. 1984. FDA study finds increase in drug use from 1971-82. Am. Pharmacy. NS24:16-18.

8. Clive, D. M., and J. F. Stoff. 1984. Renal syndromes associated with nonsteroidal antiinflammatory drugs. N. Engl. J. Med. 310:563572.

9. Hasegawa, J., P. C. Smith, and L. Z. Benet. 1982. Apparent intramolecular acyl migration of zomepirac glucuronide. Drug Metab. Dispos. 10:469-473.

10. Hijmanns van den Bergh, A. A. 1928. Der Gallenfarbstoff im Blute. J. A. Barth, Leipzig. 26-32.

11. Kuenzle, C. C., C. Maier, and J. R. Ruttner. 1966. The nature of four bilirubin fractions from serum and of three bilirubin fractions from bile. J. Lab. Clin. Med. 67:294-306.

12. Lauff, J. J., M. E. Kaspar, and R. T. Ambrose. 1981. Separation of bilirubin species in serum and bile by high performance reversedphase liquid chromatography. J. Chromatogr. 226:391-402.

13. Lauff, J. J., M. E. Kaspar, T. W. Wu, and R. T. Ambrose. 1982. Isolation and preliminary characterization of a fraction of bilirubin in serum that is firmly bound to protein. Clin. Chem. 28:629-637.

14. Lauff, J. J., M. E. Kaspar, and R. T. Ambrose. 1983. Quantitative liquid-chromatographic estimation of bilirubin species in pathological serum. Clin. Chem. 29:800-805.

15. Weiss, J. S., A. Gautam, J. J. Lauff, M. W. Sunderg, P. Jatlow, J. L. Boyer, and D. Seligson. 1983. The clinical importance of a proteinbound fraction of serum bilirubin in patients with hyperbilirubinemia. N. Engl. J. Med. 309:147-150.

16. Gautam, A., H. Seligson, E. R. Gordon, D. Seligson, and J. L. Boyer. 1984. Irreversible binding of conjugated bilirubin to albumin in cholestatic rats. J. Clin. Invest. 73:873-877.

17. McDonagh, A. F., L. A. Palma, J. J. Lauff, and T. W. Wu. 1984. Origin of mammalian biliprotein and rearrangement of bilirubin glucuronides in vivo in the rat. J. Clin. Invest. 74:763-770.

18. Smith, P. C., P. N. J. Langendijk, J. A. Bosso, and L. Z. Benet. 1985. Effect of probenecid on the formation and elimination of acyl glucuronides: studies with zomepirac. Clin. Pharmacol. Ther. 38:121127.

19. Langendijk, P. N. J., P. C. Smith, J. Hasegawa, and L. Z. Benet. 1984. Simultaneous determination of zomepirac and its major metabolite zomepirac glucuronide in human plasma and urine. J. Chromatogr. 307: 371-379.

20. Pohl, L. R., and R. V. Branchflower. 1981. Covalent binding of 
electrophilic metabolites to macromolecules. Methods Enzymol. 77:4350.

21. Stogniew, M., and C. C. Fenselau. 1982. Electrophilic reaction of acyl-linked glucuronides. Drug Metab. Dispos. 10:609-613.

22. VanBreeman, R. B., and C. C. Fenselau. 1985. Acylation of albumin by 1-O-acyl glucuronides. Drug Metab. Dispos. 13:318-320.

23. Faed, E. M. 1984. Properties of acyl glucuronides: implications for studies of the pharmacokinetics and metabolism of acidic drugs. Drug Metab. Rev. 15:1213-1249.

24. Bunn, H. F., K. H. Gabbay, and P. M. Gallop. 1978. The glycosylation of hemoglobin: relevance to diabetes mellitus. Science (Wash. DC). 200:21-27.

25. Koenig, R. J., S. H. Blobstein, and A. Cerami. 1977. Structure of carbohydrate of hemoglobin A AC. J. Biol. Chem. 252:2992-2997.

26. Higgins, P. J., and H. F. Bunn. 1981. Kinetic analysis of the nonenzymatic glycosylation of hemoglobin. J. Biol. Chem. 256:52045208.

27. Garlick, R. L., and J. S. Mazar. 1983. The principle site of nonenzymatic glycosylation of human serum albumin in vivo. J. Biol. Chem. 258:6142-6146.

28. Shaklai, N., R. L. Garlick, and H. R. Bunn. 1984. Nonenzymatic glycosylation of albumin alters its conformation and function. J. Biol. Chem. 259:3812-3817.

29. deWeck, A. L. 1983. Immunopathological mechanisms and clinical aspects of allergic reactions to drugs. In Allergic Reactions to Drugs. A. L. deWeck and H. Bundgaard, editors. Springer-Verlag, New York. 75-135.

30. Bakke, O. M., W. M. Wardell, and L. Lasagna. 1984. Drug discontinuation in the United Kingdom and the United States, 1964 to 1983: issue of safety. Clin. Pharmacol. Ther. 35:559-567. 